\title{
On the importance of the drag coefficient modelling in the double averaged Navier-Stokes equations for prediction of the roughness effects
}

\author{
F. Chedevergne ${ }^{\mathrm{a}}$ and P. Forooghi $\mathrm{b}^{\mathrm{b}, \mathrm{c}}$ \\ ${ }^{a}$ DMPE, ONERA, Université de Toulouse, F-31055 Toulouse, France ${ }^{b}$ Institute of Fluid \\ Mechanics, Karlsruhe Institute of Technology, Karlsruhe, Germany ${ }^{\mathrm{c}}$ Department of \\ Engineering, Aarhus Universiy, Aarhus, Denmark
}

\begin{abstract}
ARTICLE HISTORY
Compiled August 24, 2020

ABSTRACT

The Discrete Element Method (DEM) for modeling of flow over rough walls is revised in the framework of the DANS (Double Averaged Navier-Stokes) equations, with a special focus on the drag term appearing in the mean momentum equation as a key to the robustness of the model. A set of 14 Direct Numerical Simulations (DNS) of channel flows with systematically varying roughness topographies is considered to assess the performances of different drag coefficient closures. While the standard model of Taylor et al. [J. Fluids Eng. 107 (1985), 251257] is found not to be successful in reproducing the distribution of the drag force, a new model is derived. The new model along with the model recently proposed by Kuwata et al. [ Int. J. Heat Fluid Flow 77 (2019), 186201] are employed for the solution of channel flow along with a simple mixing length model. Both models are shown to be successful in prediction of roughness function as long as a constant in the latter model is readjusted. The velocity profiles are also well recovered and in particular the roughness sublayers are accurately reproduced.
\end{abstract}

\section{KEYWORDS}

Discrete Element Method ; Roughness modeling ; Double Averaged Navier-Stokes ; Direct Numerical Simulations

\section{Introduction}

Prediction of flow over rough surfaces is of crucial importance in various areas of engineering and geosciences. Gas turbine blades can become significantly rough due to a variety of different degradation mechanisms (deposits, erosion, corrosion, etc.) [1,2]. On the aircraft, ice accretion can lead to rough surface states [3-5]. In both cases the aerodynamic performance of the airfoil can be altered due to the presence of roughness. Similarly on ship hulls, roughness due to bio-fouling can result in performance loss [6]. Atmospheric boundary layer over city canopies and natural terrains is another area, in which prediction of flow over rough surfaces is of high importance $[7,8]$.

Different approaches can be adopted to study the flow over a rough wall using Computational Fluid Dynamics (CFD). On one side of the spectrum lie the high fidelity but computationally expensive simulations - mostly Direct Numerical Simulations

CONTACT F. Chedevergne. Email: francois.chedevergne@onera.fr 
(DNS) - in which the details of roughness geometry is fully resolved (see e.g. [9-12]). On the other side are the inexpensive approaches based on the concept of equivalent sand-grain roughness often combined with Reynolds-Averaged Navier-Stokes (RANS) models. These approaches account for the presence of roughness by modifying the wallfunctions $[13-15]$ or wall boundary condition $[16,17]$. These modifications are based on a prescribed quantity, the equivalent sand-grain roughness, that represents the effect of the considered roughness distribution. Equivalent sand-grain roughness height $k_{s}$ is defined as the size of the sand-grains in the pioneering experiments by Nikuradse [18] that produces the same friction coefficient as the surface of interest in the fully rough regime. It can be readily seen that, for an arbitrary rough surface, $k_{s}$ is not a geometrical scale but a flow property that needs to be determined based on experiments or DNS; therefore, despite being an attractive choice for commercial codes, the $k_{s}$-based models cannot be considered as purely predictive models as they rely on an a priori knowledge of $k_{s}$. Another shortcoming of these class of models is that they may not perform well in the transitionally rough regime, as $k_{s}$ is uniquely related to the skin friction only in the fully rough regime.

To reach a higher physical loyalty than what the $k_{s}$-based models can offer while avoiding the computational cost of the DNS, one can adopt an alternative strategy, in which the effect of roughness is 'modeled'. Here certain geometrical details of the roughness are given as an input to the model while an a priori knowledge of $k_{s}$ or any similar flow property is not required. A class of such models, which is widely used in the framework of boundary layer modelling, is what is often referred to as Discrete Element Method (DEM). In DEM, the roughness is represented by discrete roughness elements, whose effect is added to the spatially and temporally averaged momentum equation through a modeled closure term. The principal idea behind DEM is the Schlichtings [19] argument that the resistance of a rough surface against the flow is mainly a result of the form drag on individual roughness elements added to the viscous drag.

The DEM was first developed by Robertson [20] and Finson [21], where the drag due to the roughness elements is modelled through a sink term in the momentum equation. A constant drag coefficient $C_{d}=0.6$ was used by Finson and the system was closed using a turbulence model for the Reynolds stresses. Later, Finson [22,23] introduced a blockage coefficient $\beta$, standing for the part of the flow occupied by the roughness elements. Several contributors have brought some improvements to these pioneer works like Christoph [24,25] or Khan [26], who used mixing length to model the Reynolds stresses, or Bishnoi [27] who extended the method to the energy equation introducing a Nusselt number $N u$ similarly to the drag coefficient $C_{d}$. Lin and Bywater [28] proposed to use Žukauskas [29] data for banks of tubes in channel flows to model the drag coefficient $C_{d}$ and the Nusselt number $N u$. Finally, Taylor et al. [30] derived a set of equations from a budget analysis on a control volume that is often considered as the standard version of the DEM.

Following Lin and Bywater, Taylor et al. [30] used the form proposed by Žukauskas for the drag coefficient $C_{d}$, but the model constants were calibrated to recover experimental results [31]. The model was applied to a wide variety of boundary layer configurations [32-34]. Further improvements were proposed in the works by McClain and co-workers $[34,35]$ by adding corrections for the roughness elements with noncircular cross-sections and the existence of a dead water zone at the bottom of the roughness in dense configurations. The last point was addressed by introducing the effective height $h_{e}$ defined as the height of the roughness elements above the meltdown plane, i.e. the horizontal plane with the same height as the mean elevation of 
the surface. It is proposed that the melt-down plane should serve as the computational location of the no-slip condition in DEM simulations.

Recently, Aupoix [36] revisited the DEM and extended the range of application of the method to generic RANS solvers for any flow configuration. The derivation makes use of the volume averaging technique $[37,38]$ and leads to a set of equations close to that of the standard model but where the blockage coefficient $\beta$ directly results from the volume averaging. Similar extensions to RANS solvers were also made by Hanson et al. $[4,39]$ for ice accretation predictions and Kuwata et al. [40] for spray marine paint surfaces. The combined use of the Reynolds and volume averages give rise to the socalled DANS (Double Averaged Navier-Stokes) equations. The latter term is preferred here to designated the generalization of the DEM approach. It is worth mentioning that although inspired by the work of Taylor et al. [30] and Tarada [41] without any explicit reference to the double averaging technique, the prior work by Stripf et al [4244] proposed a similar approach for application on turbomachinery configurations.

The present work follows the lead given by Aupoix [36] and focuses on the improvement of the closure relation for the drag force appearing in the DANS equations. Our goal is to develop a robust model, which can closely predict the mean velocity profile for a wide range of of roughness topographies. The central idea of the work is to use a relation for the drag coefficient in the closure term that accurately matches the available experimental correlation proposed by Žukauskas [29]. The final model will be compared to the recent formulation given by Kuwata et al. [40,45]. Available DNS solutions for flow over roughness elements with systematically varying properties (density, slope, shape of elements) are used as a basis for the development of the model. Use of systematic DNS data particularly highlights the flexibility of the method in predicting different types of roughness with different morphological properties.

The paper is organised in the following way. First the generic DANS equations are introduced in section 2 and then applied to the channel flow configuration. The different terms requiring closure relations, in particular the drag term constituting the core of this paper, are identified. Second, section 3 presents the DNS database [46] and the mixing length models for the considered Reynolds stresses are described. Section 4 focuses on the drag coefficient modelling. A new model is discussed and compared to Kuwata et al. model [40]. Finally, section 5 offers a validation of the present approach against the DNS database. This is followed by a summary of findings in section 6 .

\section{The Double Averaged Navier-Stokes equations}

To model the effect of roughness without resolving the surrounding flow, the volumeaveraging technique $[37,47]$ is used to homogenize the problem in plane parallel to the wall. Several derivations were already realized $[36,39,45]$ in a RANS context, whereas Kuwata and Kawaguchi also applied this technique in the context of the lattice-Boltzman method [45]. Following Aupoix [36], we define $V$ the volume over which the average is taken and $V_{f}$ the part of $V$ open to the flow. The blockage coefficient $\beta$, also called plane porosity, is given by $\beta=\frac{V_{f}}{V}$. The Reynolds averaging is denoted $\bar{\square}$ while the intrinsic volume averaging is written $\langle\square\rangle^{f}$. Fluctuations around the Reynolds average and the volume average are denoted $\square^{\prime}$ and $\tilde{\square}$ respectively. 
Finally, the double-averaged momentum equations for an incompressible flow reads:

$$
\begin{aligned}
\frac{\partial \beta\langle\overline{\bar{U}}\rangle^{f}}{\partial t}+\underline{\nabla} \cdot\left(\beta\langle\underline{\bar{U}}\rangle^{f} \otimes\langle\underline{\bar{U}}\rangle^{f}\right)= & -\frac{1}{\rho} \underline{\nabla\left(\beta\langle\bar{P}\rangle^{f}\right)} \\
& \nu \underline{\nabla} \cdot\left[\overline{\left.\underline{\left(\beta\langle\bar{U}\rangle^{f}\right)}\right]}\right. \\
& -\underline{\underline{\nabla}} \cdot\left[\beta\left(\underline{\underline{\tau_{D}}}+\underline{\underline{\tau_{R}}}\right)\right]+\underline{F}
\end{aligned}
$$

$\underline{\underline{\tau_{R}}}=\underline{\nabla} \cdot\left(\beta\left\langle\underline{\bar{u}^{\prime} \otimes \underline{u^{\prime}}}\right\rangle^{f}\right)$ stands for the volume-averaged Reynolds stress tensor while $\underline{\underline{\tau_{D}}}=\underline{\nabla} \cdot\left(\beta\langle\underline{\tilde{\bar{U}}} \otimes \underline{\tilde{U}}\rangle^{f}\right)$ is the dispersive stress tensor. This term only play a role in the roughness sublayer and more specifically below the roughness crest, in the roughness wake region as shown by Forooghi et al. [12] or Jelly and Busse [48] for instance. In addition, the global contribution of the dispersive stresses to the friction is found to be weak as illustrated by Kuwata and Kawaguchi [49] through DNS of channel flows at $R e_{\tau}=600$. Therefore, following Kuwata and Kawaguchi [49], this study only models the plane-averaged drag force term and the Reynolds stresses.

An additional force $F$ appears in the momentum equations due to the homogenization process. This force stands for both the viscous and pressure drag forces acting on the roughness elements.

$$
\underline{F}=-\frac{1}{V} \int_{A_{f s}} \bar{P} n_{f s} d S+\frac{\nu}{V} \int_{S_{r}} \underline{\underline{\nabla \bar{U}}} \cdot \underline{n_{f s}} d S
$$

where $A_{f s}$ and $n_{f s}$ are the fluid/solid interface, i.e. the rough surface topology, and the associated wall normal. For the sake of clarity and compactness, in the following both averaging operators are dropped, except when needed, and we simply note $U \equiv\langle\bar{U}\rangle^{f}$.

In the present study, only channel flows are examined. The DANS equations can thus be reduced to a single non-dimensional equation:

$$
\frac{\mathrm{d}}{\mathrm{d} y^{+}}\left[\frac{\mathrm{d} \beta U^{+}}{\mathrm{d} y^{+}}-\beta \overline{u^{\prime} v^{\prime}}\right]+f_{x}^{+}+\frac{1}{h^{+}}=0
$$

where the dispersive stresses were dropped. The pressure gradient is related to the wall shear stress $\tau_{w}$ by:

$$
\tau_{w}=-\frac{\partial \beta P}{\partial x} h
$$

since the pressure gradient in the channel balances the total resistance force due to viscous and pressure efforts on the roughness. Following Forooghi et al. [46,50], $h$ is defined as the effective half-height of the channel, i.e. the half-height of the smooth channel having the same cross-section. In all the computation $h^{+} \equiv R e_{\tau}=500$.

A channel code was written to solve eq. (3) using a pseudo-unsteady approach on a very fine grid. A logarithmic distribution of 2000 points is used to discretize the wall-normal 
direction $y$. The first grid point is set to $y^{+}=0.01$ and the maximum spatial resolution obtained on the channel centerline is $\Delta y^{+}=2.7$. Considering recommendations given by McClain [35], the first grid point is then moved up to the mean roughness elevation computed as the melt-down surface. The required models for the shear stress $-\beta \overline{u^{\prime} v^{\prime}}+$ and the drag force are discussed in section 3 and 4 respectively.

Even though the derivation process vary regarding the authors $[4,45,51]$, the drag force $f_{x}^{+}$is classically modelled as:

$$
f_{x}^{+}=-(1-\beta) \frac{2}{\pi D^{+}} C_{d} U^{+^{2}}
$$

with $D=D(y)$ the local hydraulic diameter of the mean roughness element and $R e_{d}=$ $\frac{D\|U\|}{\nu}$ a characteristic local Reynolds number. Note that Hanson [52] ends up with an additional $\beta$ factor arising from the definition of the volume force $f_{x}^{+}$. Kuwata et al. [40, $45]$ provide an expression with an extra $\frac{1}{\beta}$ factor because the DANS equations (1) were previously divided by $\beta$. Nevertheless, considering circular roughness elements, the blockage factor reads $\beta=1-\frac{\pi D^{2}}{4 S^{2}}$ where $S$ is the mean spacing defined as the square root of mean area occupied by one roughness element. Introducing this definition in eq. (5), the classical formula $f_{x}^{+}=-\frac{1}{2} \frac{D^{+}}{S^{+^{2}}} C_{d} U^{+^{2}}$ proposed by Finson [21] is recovered as expected.

The solution of eq. (3) requires a model for $C_{d}$, discussed in section 4 , and a closure relation for the shear stress $-\beta \overline{u^{\prime} v^{\prime}}+$. It should be stressed that the objective of this paper is to study the effect of $C_{d}$ closure in an isolated manner from turbulence modeling. To this end we adopt a modified mixing length turbulence model, which is directly tuned to match the DNS data. This approach is also suitable for fast-paced computations of boundary layer or channel flows. Further details about the employed turbulence closure are given in section 3 .

One should add that several authors have used more elaborated RANS models suited for general purpose computations such as Stripf et al. [42,43] or Hanson et al. [39] who used variants of $k-\varepsilon$ and $k-\omega$ turbulence models respectively to complete their models for Reynolds stresses. Recently, Kuwata et al. [40] derived a general turbulence model based on a second moment closure adapted to the DANS equations. Except for Hanson et al., source terms were introduced in the turbulence models to ensure a good behavior near the wall because of the presence of roughness, especially to adapt the behavior in the roughness sublayer. In the present context such modifications would require independent validation before examining the $C_{d}$ closures. As the the present paper is focused on the drag coefficient modelling, the choice of an ad-hoc model based on mixing length concept ensures a representative model for the turbulent shear stress $-\beta{\overline{u^{\prime} v^{\prime}}}^{+}$while avoiding undesirable complications regarding turbulence modelling. Of course, the price to pay for this choice is a loss of generality in the turbulence modelling.

\section{DNS database}

Forooghi et al. [46] studied flow and heat transfer over a variety of different rough surfaces using DNS, in which the details of roughness geometry is resolved. Among others, they solved the flow over 14 roughness samples generated by discrete roughness 
elements. These 14 cases are used in the present article as the basis for calibration and examination of the modified DEM. In the following, these samples are briefly introduced; full details are available in [46].

To generate each of these roughness samples, several roughness elements are distributed randomly over a flat plate that is referred to as the 'bottom plane' hereafter. The height of elements follows a normal distribution with a mean value $k$ and a prescribed standard deviation equal to $0.28 k$. The roughness elements are axisymmetric and all the elements used in generation of a certain sample are geometrically similar. The number per unit area of the roughness elements in each sample is prescribed based on the desired mean spacing $S$, which is defined as the square root of the area occupied by one roughness element. Further details on the roughness generation algorithm can be found in [50].

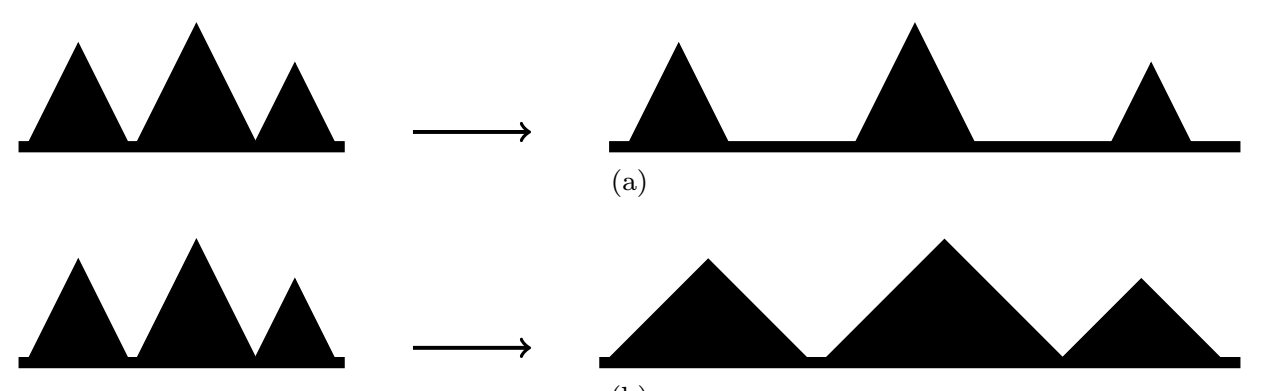

(b)

Figure 1. Schematic representation of the way the geometry of roughness samples is varied in sets A-d and Bd (a) and set A-s (b). In the former case, different roughness samples are formed by the same discrete elements (identical shape and identical size in statistical sense) but different numbers per unit area of the bottom plane. In the latter case, the geometry is scaled in wall-parallel directions, hence the shape of elements varies. In this case the slope of the elements decreases together with the number density.

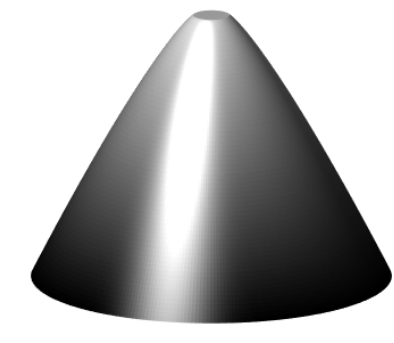

(a) type $\mathrm{A}$

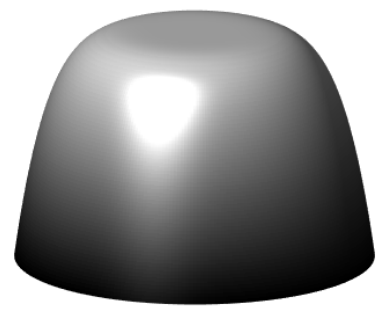

(b) type B

Figure 2. Two types of roughness elements used for generation of the samples in the A-d (a) and B-d (b) sets.

In order to systematically study the effect of morphological roughness properties, three sets of roughness samples are generated, each containing five samples. With one sample shared by two sets, it adds up to a total of 14 samples. The first two sets, labeled A-d and B-d, are used to study the effect of roughness density, the property that relates to the number of elements per unit area. To this end, while the shape, 


\begin{tabular}{l|ccccc} 
& A-d1 & A-d2 & A-d3 & A-d4 & A-d5 \\
\hline$S / k$ & 1.50 & 1.81 & 2.21 & 2.6 & 3.13 \\
$h_{e} / k$ & 0.24 & 0.16 & 0.10 & 0.08 & 0.05 \\
EES & 0.98 & 0.98 & 0.98 & 0.98 & 0.98
\end{tabular}

Table 1. Main geometrical properties of set A-d.

mean height and standard deviation of the elements are kept fixed in each set, the total number of elements are changed to create samples with different densities (see figure 1a). The difference between these two sets is in the shape of roughness elements used for each set. While all samples in A-d set are generated by elements of shape A (figure 2a), those of the B-d set have elements of shape B (figure 2b). Type A element resembles a slightly truncated cone while type B element has a flatter top and is closer to a cylinder. In both sets, the density is varied in a way that the mean spacing changes from approximately $S=1.5 k$ (the densest sample) to $3.1 k$ (the sparsest sample). Each sample in set A-d (B-d) is labeled as A-d $x(\mathrm{~B}-\mathrm{d} x)$, where $x$ is the sample ID, which progressively increases as the roughness becomes sparser.

In addition to roughness density, it is desired to systematically study the effect of roughness element slope. To do so, another set of samples, labeled A-s, is generated. Here a certain roughness geometry is up-scaled in wall-parallel directions (in other words it is stretched horizontally) as schematically shown in figure 1b. By doing so, the roughness elements are deformed and their slope decreases progressively. Each sample in this set is labeled A-s $x$ where the ID $x$ increases as the slope decreases. The first sample in this set (A-s1) is chosen to be identical to sample A-d1. To measure the variation in the slope of the elements we define the parameter 'effective element slope' (EES) as follows:

$$
\mathrm{EES}=\frac{2 S_{f}}{S_{w}}
$$

where $S_{f}$ and $S_{w}$ are the projected frontal area and the wall-parallel projected area of a single element. It can be shown that EES is equal to the mean streamwise gradient of the element surface height.

As stated by McClain et al. [35], the mean elevation play a significant role in dense configuration. The underlying physical phenomenon at play is the existence of thin separation zones behind roughness that do not contribute to friction increase. Consistently with Forooghi et al. [53], the mean elevation is defined here as the ratio of the volume of the mean roughness $V$ over the mean area occupied by this roughness element.

$$
h_{e}=\frac{V}{S^{2}}
$$

Tables 1-3 summarize the morphological properties of the samples used in this study. Note that samples A-d1 and A-s1 are identical.

Details of the numerical configuration adopted in DNS is given in [46]. Briefly, the simulations are carried out in a cubic computational domain with periodic boundary conditions in the streamwise and spanwise directions, which corresponds to a fully developed channel flow. The roughness elements are mounted on the lower boundary 


\begin{tabular}{l|ccccc} 
& B-d1 & B-d2 & B-d3 & B-d4 & B-d5 \\
\hline$S / k$ & 1.55 & 1.81 & 2.21 & 2.6 & 3.13 \\
$h_{e} / k$ & 0.19 & 0.14 & 0.09 & 0.07 & 0.05 \\
EES & 2.04 & 2.04 & 2.04 & 2.04 & 2.04
\end{tabular}

Table 2. Main geometrical properties of set B-d.

\begin{tabular}{l|ccccc} 
& A-s1 & A-s2 & A-s3 & A-s4 & A-s5 \\
\hline$S / k$ & 1.50 & 1.92 & 2.83 & 3.9 & 5.65 \\
$h_{e} / k$ & 0.24 & 0.24 & 0.24 & 0.24 & 0.24 \\
EES & 0.98 & 0.76 & 0.52 & 0.37 & 0.26
\end{tabular}

Table 3. Main geometrical properties of set A-s.

of the domain (bottom plane). For the upper boundary, shear-free boundary condition is applied. This configuration is often referred to as open channel in the context of DNS. All simulations used in the present article are done at friction Reynolds number of approximately 500, and mean roughness element size in wall units is equal to $k^{+}=65$ in all cases. Governing equations are solved using the pseudo-spectral solver SIMSON [54], and the roughness is captured using the immersed boundary method based on [55].

The DNS database can be used to provide guidance for modelling. Introducing DNS profiles in eq. (3), distributions of drag coefficient $C_{d}$ across the channel can be estimated. These profiles will be used in section 4 to assess different models for $C_{d}$. Similarly, mixing lenghts distributions can be obtained from the DNS profiles to help closing eq. (3). In particular a model for the shear stress $-\beta{\overline{u^{\prime} v^{\prime}}}^{+}$is required and can be deduced from the DNS results. In addition, ${\overline{u^{\prime 2}}}^{+}$may also be considered for modelling the drag coefficient $C_{d}$ (see eq. (12) in section 4). Therefore, a model is needed too. The corresponding mixing lengths $l_{u v}$ and $l_{u u}$ are found by considering that the following relations hold:

$$
\begin{aligned}
-\beta{\overline{u^{\prime} v^{\prime}}}^{+} & =l_{u v}^{2}\left(\frac{\partial \beta U^{+}}{\partial y^{+}}\right)^{2} \\
\beta{\overline{u^{\prime 2}}}^{+} & =l_{u u}^{2}\left(\frac{\partial \beta U^{+}}{\partial y^{+}}\right)^{2}
\end{aligned}
$$

In the above relations, Prandtl mixing length hypothesis is invoked for both Reynolds stresses while considering a unique characteristic timescale based on the velocity gradient $\frac{\partial \beta U^{+}}{\partial y^{+}}$. The $\beta$ factor is not required for ${\overline{u^{\prime 2}}}^{+}$but is added for the sake of homogeneity regarding the definition of Reynolds stresses $\underline{\underline{\tau_{R}}}$. Note that the DNS statistics were such that only the total stress tensor $\underline{\underline{\tau_{D}}}+\underline{\underline{\tau_{R}}}$ was recorded. As a consequence, the mixing length $l$ is deduced from the total shear stress including the effect of the dispersive stresses. From Jelly and Busse [48] or Forooghi [56], it was proved that the dispersive stresses are an order of magnitude lower than the Reynolds stresses and their are restricted to the roughness sublayer.

The model for $l_{u v}$ is directly derived from the standard mixing length formulation applicable to channel flows with smooth wall from Nikuradse $[18,57]$ while the model 
for $l_{u u}$ is deduced from the observation of the DNS data. Note that the final model for $l_{u u}$ provides a plateau region in the logarithmic region as expected for channel flows at large Re number. However, to account for the presence of the roughness and in accordance with the recovery of the logarithmic region, distributions must be shifted using the wall correction $\varepsilon^{+}[58,59]$. Jackson [60] showed that $\varepsilon$ corresponds to the height where the averaged drag force acts but in the present study an analytical formula is retained which reads:

$$
\frac{\varepsilon}{k}=0.45+\frac{0.04}{E E S}
$$

It gives values closed to those adopted by Squire et al. [59], i.e. $\frac{\varepsilon}{k}=0.5$, and allows to account for the effect on the effective slope on the point of application of the drag force. Similar approaches are also encountered to model roughness effects on sand ripples or dunes [61,62].

To extend the models for $l_{u v}$ and $l_{u u}$ down to the wall, hyperbolic tangent functions are adopted to obtain the desire behaviors below the roughness crest. Hyperbolic functions are convenient from the numerical side since they are infinitely differentiable. In addition, in the present context with the available DNS data, these functions allow a sufficient representation of the near wall behaviors. Finally, we retain:

$$
\begin{aligned}
& f_{u v}(y)=h\left(0.14-0.08\left(1-\frac{y}{h}\right)^{2}-0.06\left(1-\frac{y}{h}\right)^{4}\right) \\
& f_{u u}(y)=\kappa y \\
& l_{u v}=\max \left[f_{u v}(y-\varepsilon), f_{u v}(k-\varepsilon) \tanh \left(4 \frac{y}{k}\right)\right] \\
& l_{u u}=\max \left[f_{u u}(y-k)+3 \beta f_{u u}(k-\varepsilon), 3 \beta f_{u u}(k-\varepsilon) \tanh \left(4 \frac{y}{k}\right)\right]
\end{aligned}
$$

Comparisons between the mixing lengths deduced from the DNS database and the models given by eq. (10) are drawn on figure 3 for four representative cases. Satisfactory agreements are obtained with the modelled mixing lengths despite discrepancies below the roughness crest and near the channel centerline. Variations of $l_{u v}$ and $l_{u u}$ in the DNS near the channel centerline are the manifestation of the influence of the symmetry condition on the $U^{+}$profile generating small riddles. The so-called open channel condition is used by Forooghi et al. to reduce the computational cost but fluctuations profiles are slightly affected by this condition around the centerline [53]. As claimed by the authors, these variations are not detrimental regarding the region of interest, i.e. the near-wall region. As a consequence, there is no need for the modelling of the mixing lengths do reproduce these behaviors near the channel centerline. In contrast, differences observed near $y=0$ may be more prejudicial. This zone is governed by the competition between the drag force and the divergence of the total stress tensor. Therefore, in section 5, an assessment of this mixing length model is made to justify its use in the present context. 


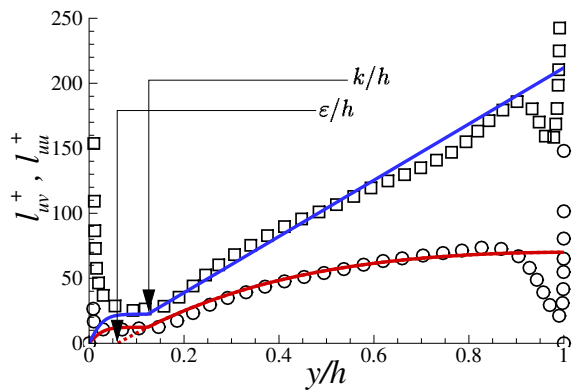

(a) A-d1

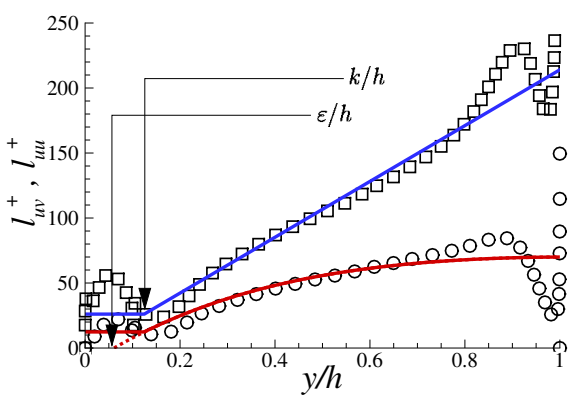

(c) B-d1

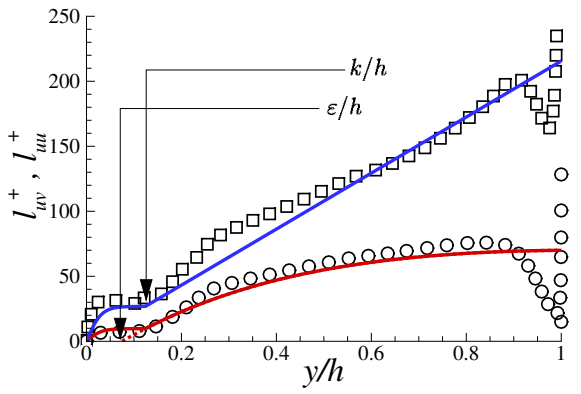

(b) A-s5

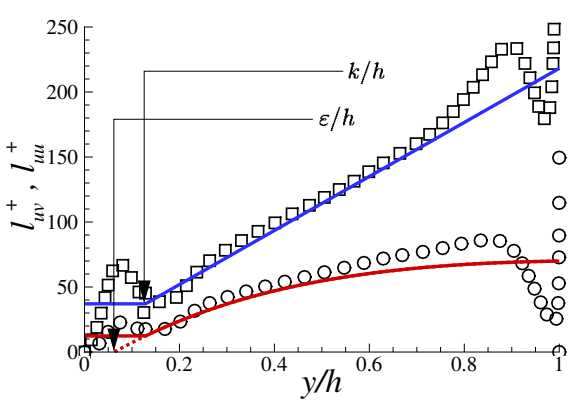

(d) B-d5

Figure 3. Distributions of the mixing lenghts defined by eq. (8). The black symbols $\circ$ and $\square$ stands for the DNS results for $l_{u v}$ and $l_{u u}$ respectively. The red and blue solid lines are the models given by eq. (10) for $l_{u v}$ and $l_{u u}$ respectively.

\section{Drag coefficient modelling}

The closure of eq. (3) requires a model for the drag coefficient $C_{d}$ in order to compute the drag force (5). The most commonly adopted model for $C_{d}$ was derived by Taylor et al. [30] and was calibrated on Schlichting's experiments [19,63] revisited by Coleman et al. [31]. The main idea behind this model lies in the work of Žukauskas [29] for bank of tubes placed in channel flows. These data correlate the drag coefficient $C_{d}$ to the Reynolds number based on the diameter of the tubes $R e_{d}$. The final model reads:

$$
\begin{cases}C_{d}=\left(\frac{R e_{d}}{1000}\right)^{-0,125} & R e_{d} \leq 60000 \\ C_{d}=0.6 & R e_{d}>60000\end{cases}
$$

Hanson et al. used the same drag coefficient model for their computations [4,39].

Similarly to models derived for porous media where a linear and quadratic dependence on the velocity are accounted for, Kuwata et al. $[40,45]$ proposed to write the drag coefficient as:

$$
C_{d}=\frac{C_{1}}{\beta R e_{d}}+\frac{C_{2}}{\beta^{3 / 2}}\left(1+\frac{\overline{u^{\prime 2}}}{\|U\|^{2}}\right)
$$


Factors $\beta$ and $\beta^{3 / 2}$ are corrections functions introduced to mimic the mutual dependencies between roughness due to sheltering in dense configurations. Constant $C_{1}$ and $C_{2}$ equal 71 and 0.79 respectively. The idea of using linear and quadratic terms for calculation of drag has been proposed for the first time in the framework of parametric forcing models by Forooghi et al. [56].

Before evaluating these models against the DNS data, a last model can be derived. Unlike Taylor et al. [30] who calibrated their model on a set of experiments, the raw data of Žukauskas [29] are considered to derive a new model for $C_{d}$. Žukauskas proved that the pressure drop due to the tubes in the channel is related to the Reynolds number $R e_{d}$ and also to the arrangement of the tubes. For the present models, only the staggered configurations are retained and figure 64 of Žukauskas's paper [29] is analysed. A fitting function is utilized to reproduce the data collected by Žukauskas and the final expressions read:

$$
\begin{aligned}
\log \xi & =(0.58 f-0.86) \log \beta R e_{d}+1.82-1.1 f & \beta R e_{d} \leq 116883 \\
\xi & =0.2 & \beta R e_{d} \geq 116883
\end{aligned}
$$

where the blending function $f$ is:

$$
\begin{aligned}
f & =0 & \beta R e_{d} & \leq 60 \\
f & =1-\frac{60}{\beta R e_{d}} & 60 \leq \beta R e_{d} & \leq 200 \\
f & =1-\frac{1}{\left(\frac{\beta R e_{d}}{10}\right)^{0,4}+\left(\frac{\beta R e_{d}}{10000}\right)^{2,78}} & 200 \leq \beta R e_{d} & \leq 116883
\end{aligned}
$$

The resulting model for $C_{d}$ is:

$$
C_{d}=3 \frac{\xi}{\beta^{4}}
$$

It can be readily seen that in eq. (15), referred to as the present model in all the following, the closure term depends not only on $R e_{d}$ but also on $\beta$ contrary to the standard model (11) given by Taylor et al. [30]. Remark that the Reynolds number used in eq. (13) is $\beta R e_{d}$, i.e. it is build on the volume averaged velocity $\langle U\rangle$ and not $\langle U\rangle^{f}$. The dependence on $\beta$ is a result of the influence on the tube arrangement in the data of Žukauskas. More precisely, the drag coefficient depends on the spacing between tubes and it is accounted for through the blockage factor $\beta$. A similar dependency is also made in the model derived by Kuwata et al. (12).

As mentioned herein above, drag coefficient distributions are extracted from the DNS profiles considering the balance given in eq. (3). Comparisons against the models exposed above are given on figure $4 \mathrm{a}$ and $4 \mathrm{c}$ for two representative cases. First of all, the standard model (11) provides manifestly drag coefficients several orders of magnitude lower than those exhibited by the DNS. The success of the whole model initiated by Taylor et al. and further improved by McClain [34,35] results from the competition between this drag coefficient model and the retained mixing length model used for the shear stress. The model is similar to the one used in smooth configuration and rely on the Van Driest damping function. The same is true for results obtained 


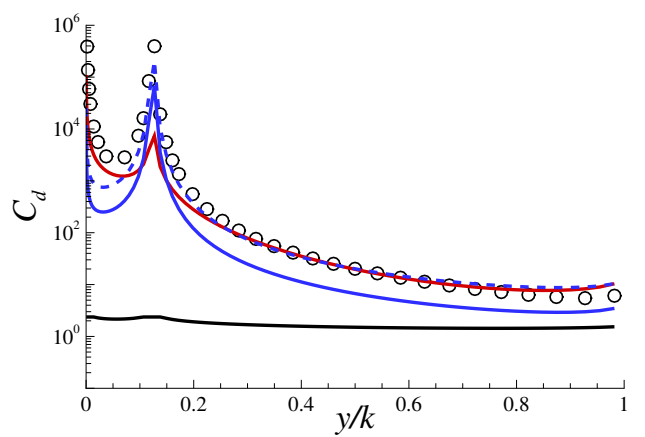

(a) A-d1

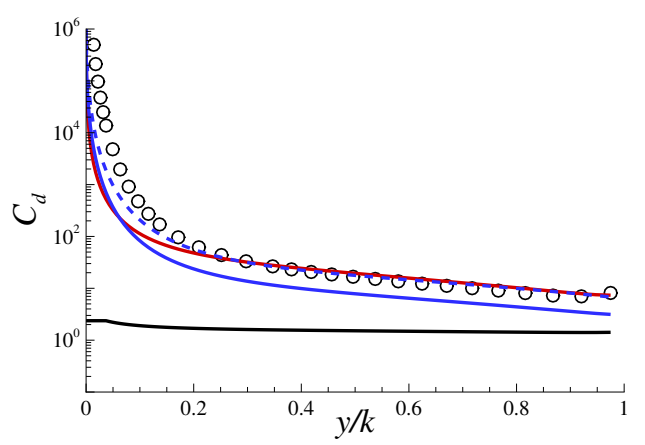

(c) B-d1

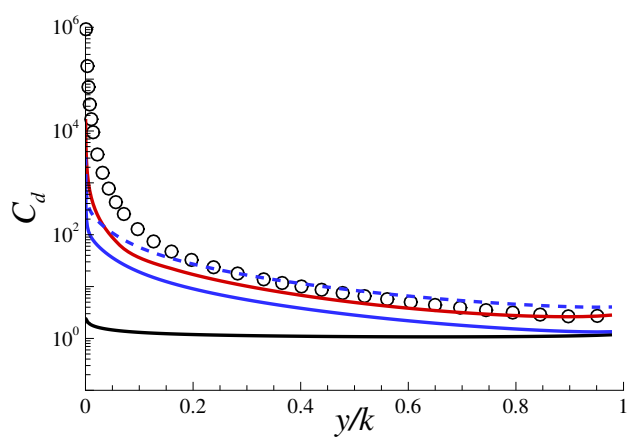

(b) A-s5

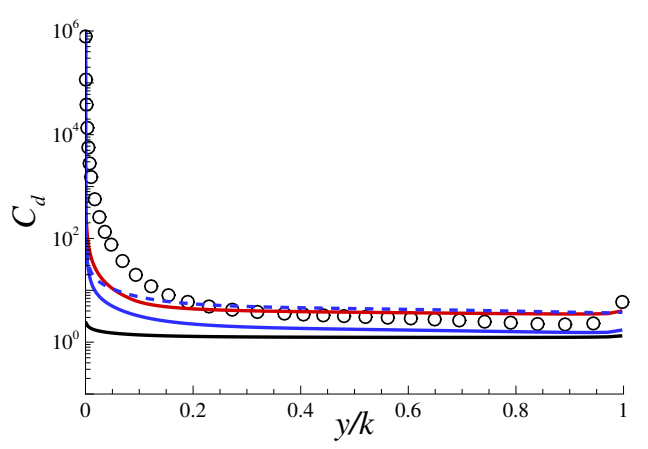

(d) B-d5

Figure 4. Drag coefficient distributions along the roughness height for different configurations. Black symbols $\circ$ are DNS results, black solid line corresponds to the standard model of eq. (11), blue solid and dashed lines stand for Kuwata et al. model of eq. (12) with the nominal $C_{2}$ value $\left(C_{2}=0.79\right)$ and the modified $C_{2}$ constant $\left(C_{2}=2.37\right)$ respectively and the red solid line is the present model given by eq. (15).

by Hanson and Kinzel, who used the standard drag model of eq. (11) together with the $k-\omega$ SST turbulence model without any specific modification. Regarding the present DNS results of Forooghi et al. [46], using the model of eq. (11) in conjunction with the mixing length model given by eq. (10) yields erroneous results and does not allow to recover the roughness effect observed in the DNS. For this reason, the standard model for $C d$ is no longer discussed in the rest of this paper.

On figure $4 \mathrm{a}$ a peak is observed near $\frac{y}{k}=0.18$. It is related to the velocity profiles $U^{+}$that exhibits negative values for $y^{+} \in\left[0,0.18 k^{+}\right]$. Surprisingly, Case B-d1 does not show the same behavior whereas the slope of the roughness elements is steeper $(E E S=2.04)$ and is thus expected to induce larger seperations. DNS results indicate that fluid is by-passing the roughness element near the wall which results in a double-averaged velocity profile $U^{+}$without recirculating zone.

The drag coefficient model developed by Kuwata et al. and given by eq. (12) behaves correctly but with lower amplitude levels than those of the DNS. The problem comes from the quadratic term whose contribution is too weak. Changing coefficient $C_{2}$ to 2.37 enables the model to reasonably fit the DNS results for all configurations as illustrated on figure 4 for cases $\mathrm{A}-\mathrm{d} 1$ and $\mathrm{B}-\mathrm{d} 1$. In the following, the model of Kuwata et al. will be used considering $C_{1}=71$ and $C_{2}=2.37$.

The drag modelling proposed in eq. (15) also enables to recover the distributions obtained in the DNS. A very satisfactory agreement is found on all the configurations 
and the distributions of $C_{d}$ nicely compare with those of Kuwata et al. model. Yet the two models rely on very different bases. Kuwata et al. based their model on a separation of $C_{d}$ between viscous and inertial effects that finally ends in a formulation similar to the Darcy-Forchheimer law. One of the advantages of eq. (12) is thus to be able to distinguish linear and quadratic velocity contributions and to isolate the contribution of the Reynolds stresses. On the opposite, all these contributions are gathered within the correlation extracted from Žukauskas data in eq. (15). Conversely, geometry dependent factors $\beta$ and $\beta^{3 / 2}$ were introduced in eq. (12) out of necessity while no additional geometrical correction is required for the present model of eq. (15). At last, it must be pointed out that coefficient $C_{2}$ of eq. (12) had to be adjusted to fit the DNS data whereas no calibration was required when using the present model of eq. (15). Although the approaches to develop those two models completely differ, the main dependencies on the Reynolds number and on the roughness topology are well reproduced in both expressions, as proved in figure 4. These two models, combined with the closure relation for the shear stress (10), were implemented in the channel code mentioned in section 2 .

It is worth mentioning that the recourse to the mean elevation can be avoided using a modified $C_{d}$ evolution at very low Reynolds number $R e_{d}$. Despite the merit of the mean elevation to account for the existence of a recirculation zone in the very near wall region, a more faithful physical model should not be using such an artefact. One possible route to follow is to drastically increase $C_{d}$ as $R e_{d}$ tends zero, thereby forcing $U$ to become zero. This evolution is neither included in the model of Kuwata et al. (12) nor in the present model (15) derived from Žukauskas database [29]. For the latter, given that the correlation for $C_{d}$ is obtained from channel flows with banks of tubes, it is obvious that the desired effect can not be included in the present form. Such a modification has not been tested here but can be easily made by changing some of the constants in eq. (13).

\section{Validation}

The 14 cases of the DNS database were computed with the DANS channel code described in section 2. For each case, two computations were realized using either the drag coefficient model of Kuwata et al. and given by eq. (12) or the present model of eq. (15).

Before examining the velocity profiles obtained with the two models of eq. (12) and eq. (15) retained for the drag coefficient $C_{d}$, the model used for the shear stress tensor is assessed against the DNS data.

\subsection{Shear stress profiles}

Figures $5 \mathrm{a}$ and $5 \mathrm{~b}$ plot the distributions of the total shear stress provided by the DNS and the computations using the DANS approach in the channel code. It is reminded that the DNS total shear stress contain the dispersive stresses whereas formally there are set to zero in the present DANS approach. But, the mixing lenght model (10) used was build from the DNS data, i.e. the total shear. In other words, the Reynolds shear stress model used in the DANS computations include the effect of the dispersive shear stress. Figures clearly indicate that the standard mixing length model of Nikuradse works well above the roughness sublayer $y / k>3-5$. However, due to the absence 


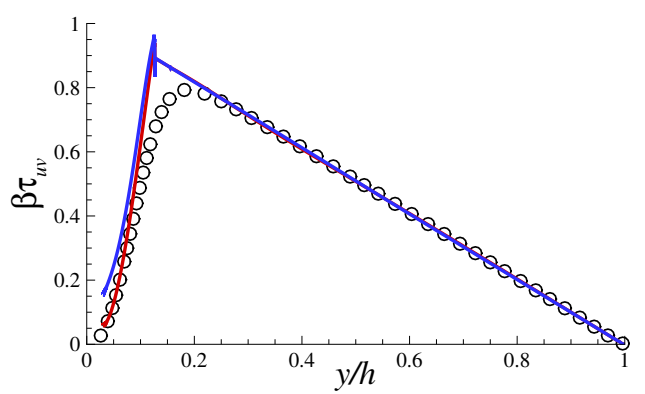

(a) A-d1

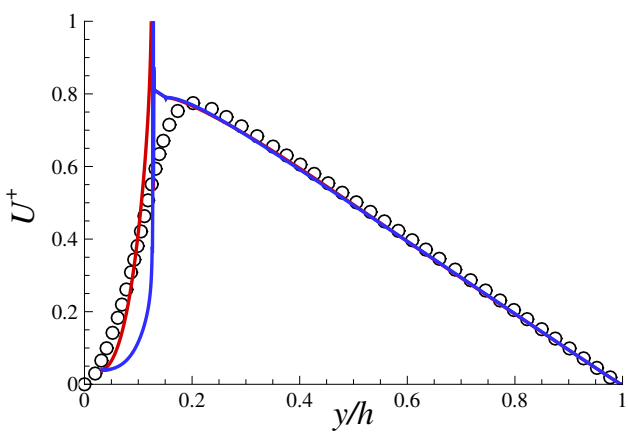

(b) B-d1

Figure 5. Total shear stress profiles across the open-channel. Black symbols $\circ$ are the DNS results, the blue solid lines are the DANS computed profiles obtained with the drag coefficient model $C_{d}$ of eq. (12) and the red solid lines are those obtained with the present $C_{d}$ model given by eq. (15).

of diffusion for the turbulent model the peak for $\beta \tau_{u v}$ is located at $y=k$ where the $C_{d}$ coefficient becomes zero. The non-continuous nature of the mixing lenght model due to the derivation of $\beta$ also contributes to reinforce the peak amplitude. The more elaborated RSM model of Kuwata et al. [40] has not this restriction since transport equations including diffusion terms are considered for the turbulence model.

The differences observed between the two computations below the roughness crest are due to the influence of the drag modelling. Case A-d1 shows a good and almost similar behavior for the two computations whereas case B-d1 was deliberately pushed to the forefront to illustrate a worse behavior of the $C_{d}$ model coming from Kuwata et al. Remark that case B-d1 is the unique exception among the 14 tested cases where the formulation of eq. (12) for $C_{d}$ drastically departs from that of eq. (15). Further analysis showed that the contribution of the term $\frac{\overline{u^{\prime 2}}}{\|U\|^{2}}$ is too high and that $C_{2}$ coefficient should be separated into two independent coefficients for the mean and fluctuating quadratic contributions.

Although a simple algebraic turbulence modelling (10) was used in the DANS computations, the obtained shear stress distributions are satisfactory in the present context of channel flows with rough walls. Moreover, even if limited, this model is sufficient to differentiate the drag coefficient models as expected.

\subsection{Velocity profiles}

Six illustrative examples of velocity profiles resulting from the DANS computations are shwon on figure 6 . The computed profiles are compared to those provided by the DNS database of Forooghi [46]. Linear and logarithmic scales are used for the wallnormal coordinate to highlight different points. On each figure, the equivalent sand grain height $k_{s}$ corresponding to the DNS profile was reported. The determination of $k_{s}$ is made from the following considerations. According to Perry et al. [64] or Raupach [58], the velocity profile $U^{+}$over a rough surface can be written under the form $U^{+}=\frac{1}{\kappa} \ln \left(y^{+}-\varepsilon^{+}\right)+C-\Delta U^{+}$, where $C$ is a constant valid under smooth conditions, $\Delta U^{+}$is the roughness function and $\varepsilon^{+}$the wall shift enabling to recover the logarithmic region and previously discussed in section 3. Considering Nikuradse's 


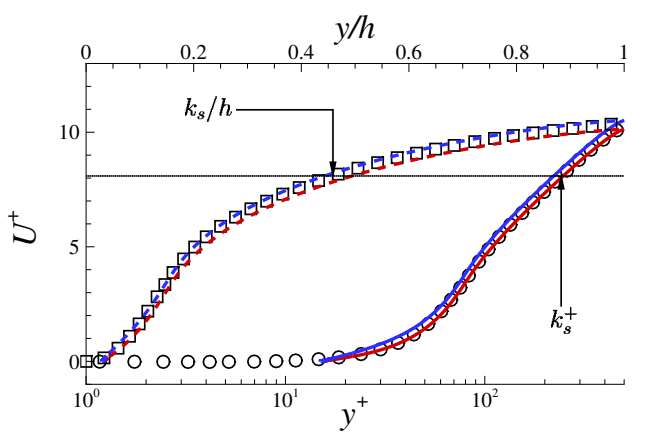

(a) A-d1

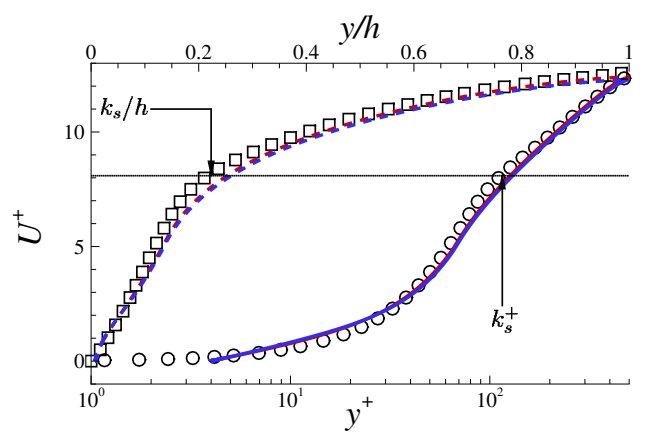

(c) A-s5

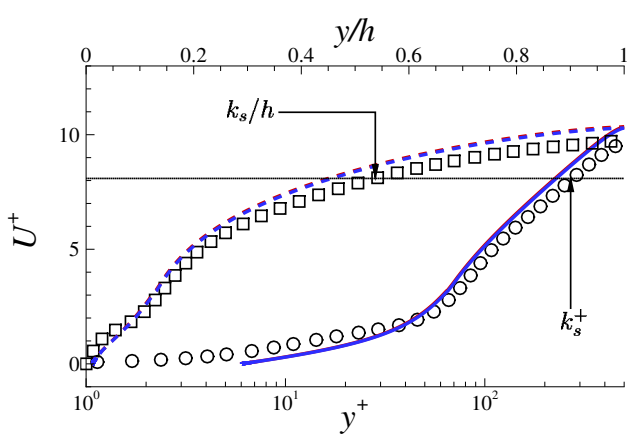

(e) B-d3

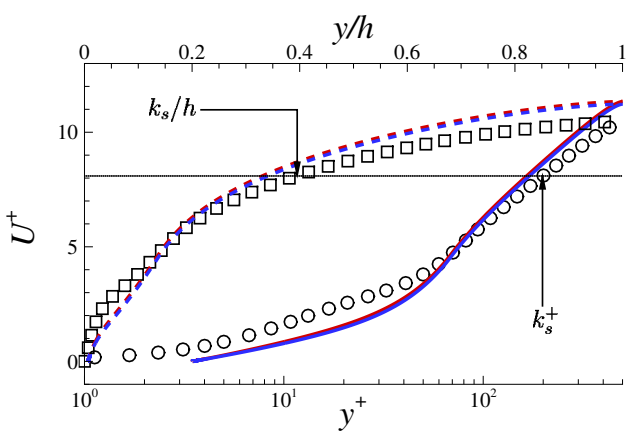

(b) A-d5

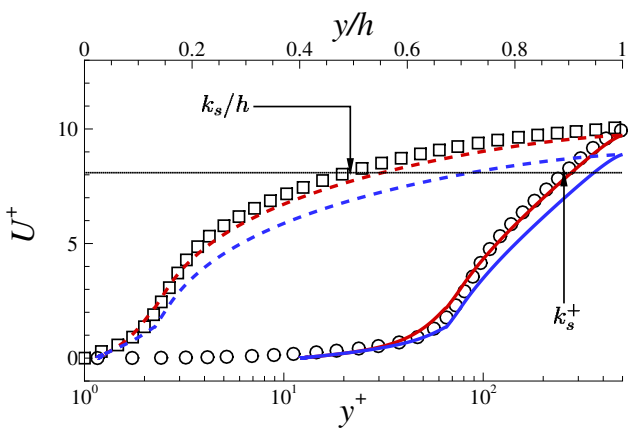

(d) B-d1

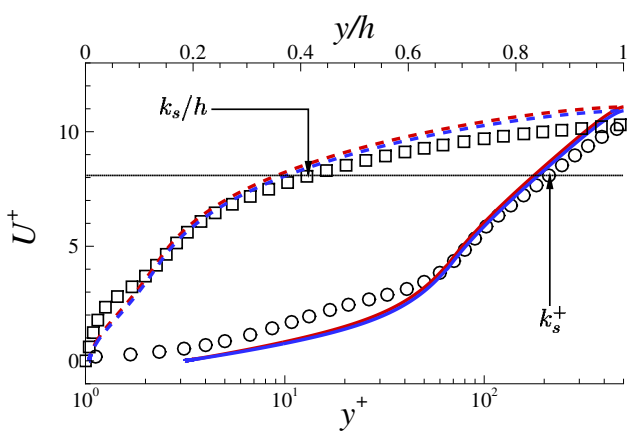

(f) B-d5

Figure 6. Velocity profiles across the open-channel. Black symbols o are the DNS profiles plotted in semilogarithmic scale and $\square$ symbols shows the DNS profiles in linear scale. The red and blue solid lines represent the DANS profiles in semi-logarithmic scales obtained from the present $C_{d}$ model - eq. (15) - and that of eq. (12), respectively. The corresponding profiles plotted in linear scale are respectively given by the blue and red dashed lines.

relation for the roughness function in the fully rough regime $\Delta U^{+}=\frac{1}{\kappa} \ln k_{s}^{+}+C-B$, with $B=8.48$, trivial manipulations provides $U^{+}\left(k_{s}^{+}\right)=\frac{1}{\kappa} \ln \left(1-\frac{\varepsilon^{+}}{k_{s}^{+}}\right)+B$. For all the configurations, given the expression (9) for $\varepsilon^{+}$, one finds $U^{+}\left(k_{s}^{+}\right)=8.09$ since for all cases we have $\frac{\varepsilon^{+}}{k_{s}^{+}} \approx 0.15$. Therefore, plotting the horizontal line $U^{+}=8.09$ on figures $6 \mathrm{a}$ to $6 \mathrm{f}$ allows to easily find the corresponding $k_{s}^{+}$values. Values obtained using this simple method are very closed to those found by Forooghi [46] within a 
few percents. On figures 6 a to $6 \mathrm{c}$, the effects of a drop of density or element slope are clearly visible on $k_{s}$ and are pretty well recovered by the DANS computations. Both drag coefficients models provide the good trends even if they tend to underestimate the roughness function $\Delta U^{+}$when dropping the roughness element density in figure 6b. As alluded ealier, constant $C_{2}$ in Kuwata et al. model was changed to $C_{2}=2,37$.

Figure $6 \mathrm{~d}$ illustrates again the fact that the $C_{d}$ model inspired from the work by Kuwata et al. produces too much drag and eventually generates too large roughness function $\Delta U^{+}$. This behavior does not occur for cases B-d2 to B-d5 as shown on figures $6 \mathrm{e}$ and $6 \mathrm{f}$. The present model for $C_{d}$ derived from Žukauskas database allows a good restitution of the roughness function for all computed cases.

One of the achievement of the present DANS formulation using either the drag coefficient modelling of eq. (12) or of eq. (15) is the ability to faithfully describe the velocity profile $U^{+}$all over the roughness sublayer. Previous formulation relying on the standard $C_{d}$ models (11) were unable to recover this part of the velocity profile. Taking a close look to the results for all computed cases does not clearly allow to discriminate between the two retained models for $C_{d}$. Except for case B-d1, the two models provides very satisfactory results. Each of these two $C_{d}$ models have their own advantages and more validation test cases may be required to be able to differentiate these two approaches.

\section{Conclusion}

The DANS equations were used in a channel flow configuration to assess the performances of different drag coefficient models in the framework of DEM for modeling of flow over rough walls. A set of 14 DNS cases [46] were used as the benchmark to perform this evaluation. Simple closure relations were used for the Reynolds stresses based on the mixing length concept. It was shown that the standard $C_{d}$ model of eq. (11) provided by Taylor et al., and generally considered as the mainstream model, is not able to reproduce the drag force term extracted from the DNS database. The relative success of this model is likely related to the errors due to the drag and turbulence models canceling out. In the present work, a new model, directly derived from the Žukauskas experimental work, was developed - eq. (15). The model recently proposed by Kuwata et al. [40] - eq. (12) - was also assessed in the present work as a reference model. Our developed channel code provided very satisfactory results for the mean velocity profiles compared to DNS data using both the present $C_{d}$ closures and that proposed by Kuwata et al.. In the latter case, the model constant $C_{2}$ had to be readjusted. In particular, the roughness sublayers, extended up to about $3 k_{s}$, are well captured for all the considered configurations, including slope and density variations of the roughness distribution. Except for one of the test cases (B-d1), where the model given by eq. (12) does not reproduce the DNS profiles satisfactorily, both models for the drag coefficient $C_{d}$ perform reasonably well and offer encouraging alternatives to the standard model.

Further extension of the present model beyound the $C_{d}$ closure are intended in future to account for roughness effects in non-canonical flows. To this end, a more universal turbulence model is required. While the 7-equation Reynolds Stress Model proposed by Kuwata et al. [40] can be considered as the most advanced model in the framework of roughness modeling, a simpler model is desired in an industrial context. A possibility is to include certain aspects of Kuwata et al. model in a 2-equation model such as the $k-\varepsilon$ based model of Stripf et al. $[42,43]$ or the $k-\omega$ SST based model of 
Hanson et al. [39]. In particular, from the DANS momemtum equation, the Reynolds stress equations were (re)derived to formally identified the drag-like terms involved these equations. Complementary, the similar term is introduced in the dissipation equation to close the turbulence model and obtained the desired behavior in the near wall region. The same can be done for 2-equation models to provide adapted turbulent closures. Finally, an extension of the present DANS approach to include the energy equation seems necessary since thermal constraints are of crucial importance for most roughness-related applications. Once again, the experiments of Žukauska may serve as the basis for development of the closure relation accounting for heat transfer at the fluid-solid interface.

\section{Acknowledgement(s)}

PF gratefully acknowledges financial support from German Research Foundation (DFG) through the project SFB/TRR 150 for this research.

\section{References}

[1] Bons J, Taylor R, McClain S, et al. The many faces of turbine surface roughness. Journal of Turbomachinery. 2001 October;123:739-748.

[2] Bons J. A review of surface roughness effects in gas turbines. Journal of Turbomachinery. 2010 April;132:021004-1-16.

[3] Dukhan N, Masiulaniec K, De Witt K, et al. Experimental heat transfer coefficients from ice-roughened surfaces for air deicing design. Journal of Aircraft. 1999 NovemberDecember;36(6):948-956.

[4] Hanson D, Kinzel M. Application of the discrete element method to ice accretion geometries. In: 46th AIAA Fluid Dynamics Conference; jun. American Institute of Aeronautics and Astronautics; 2016. Available from: https://doi.org/10.2514/6.2016-4109.

[5] McClain S, Tin̆o P, Kreeger R. Ice shape characterization using self-organizing maps. Journal of Aircraft. 2011 March-April;48(2):724-729.

[6] Schultz MP. Effects of coating roughness and biofouling on ship resistance and powering. Biofouling. 2007;23(5):331-341.

[7] Raupach M. Simplified expressions for vegetation roughness length and zero-plane displacement as functions of canopy height and area index. Boundary-Layer Meteorology. 1994;71(1-2):211-216.

[8] Anderson W, Meneveau C. Dynamic roughness model for large-eddy simulation of turbulent flow over multiscale, fractal-like rough surfaces. Journal of Fluid Mechanics. 2011; 679:288-314.

[9] Orlandi P, Leonardi S. DNS of turbulent channel flows with two-and three-dimensional roughness. Journal of Turbulence. 2006;(7):N73.

[10] Cardillo J, Araya G, Newman J, et al. DNS of a turbulent boundary layer with surface roughness. Journal of Fluid Mechanics. 2013;729:603-637.

[11] Chan L, MacDonald M, Chung D, et al. A systematic investigation of roughness height and wavelength in turbulent pipe flow in the transitionally rough regime. Journal of Fluid Mechanics. 2015;771:743-777.

[12] Forooghi P, Stroh A, Schlatter P, et al. Direct numerical simulation of flow over dissimilar, randomly distributed roughness elements: A systematic study on the effect of surface morphology on turbulence. Physical Review Fluids. 2018;3(4):044605.

[13] Blocken B, Stathopoulos T, Carmeliet J. Cfd simulation of the atmospheric boundary layer: wall function problems. Atmospheric environment. 2007;41(2):238-252. 
[14] Suga K, Craft T, Iacovides H. An analytical wall-function for turbulent flows and heat transfer over rough walls. International Journal of Heat and Fluid Flows. 2006 October; $27(5): 852-866$.

[15] Chedevergne F. Analytical wall function including roughness corrections. International Journal of Heat and Fluid Flow. 2018 oct;73:258-269.

[16] Aupoix B, Spalart P. Extensions of the spalart-allmaras turbulence model to account for wall roughness. International Journal of Heat and Fluid Flow. 2003;24(4):454-462.

[17] Aupoix B. Roughness corrections for the $\mathrm{k}-\omega$ shear stress transport model: Status and proposals. Journal of Fluids Engineering. 2014 Sep;137(2). Available from: https://doi.org/10.1115/1.4028122.

[18] Nikuradse J. Strömungsgesetze in rauhen Rohren. VDI-Forschungsheft; 1933. 361.

[19] Schlichting H. Experimentelle Untersuchungen zum Rauhigkeitsproblem. Ingenieur Archiv. 1936 February;VII(1):1-34.

[20] Robertson J. Surface resistance as a function of the concentration and size of roughness elements [dissertation]. State University of Iowa; 1961.

[21] Finson M. A Reynolds stress model for boundary layer transition with application to rough surfaces. Wakefield, Massachusetts: Physical Sciences Inc. 1975. Interim scientific report.

[22] Finson M, Clarke A. The effect of surface roughness character on turbulent reentry heating [Aiaa paper 80-1459 $15^{\text {th }}$ thermophysics conference, snowmass, colorado]; 1980.

[23] Finson M. A model for rough wall turbulent heating and skin friction [Aiaa paper 82-0199 $20^{\text {th }}$ aerospace science meeting, orlando, florida]; 1982.

[24] Christoph G, Pletcher R. Predictions of rough-wall skin friction and heat transfer. AIAA Journal. 1983 April;21(4):509-515.

[25] Christoph G, Fiore A. Experimental and computational study of roughness effects at $\mathrm{M}=6$ [Aiaa paper 84-1681 $17^{\text {th }}$ fluid dynamics, plasma dynamics and laser conference, snowmass, colorado]; 1984.

[26] Khan Z. An analytical study of the effects of surface roughness on a compressible turbulent boundary layer [master's thesis]. Wright-Patterson Air Force Base, Ohio: Air Force Institute of Technology; 1983.

[27] Bishnoi P. Computation of skin friction and heat transfer with inclusion of stagnation heating of roughness elements for turbulent boundary layer flows [Aiaa paper 88-0175 $26^{\text {th }}$ aerospace sciences meeting, reno, nevada]; 1988.

[28] Lin T, Bywater R. Turbulence models for high-speed, rough-wall boundary layers. AIAA Journal. 1982 March;20(3):325-333.

[29] Z̆ukauskas A. Heat transfer from tubes in crossflow. (Advances in Heat Transfer; Vol. 8). J.P. Hartnett and T.F. Irvine; 1972. p. 93-160.

[30] Taylor R, Coleman H, Hodge B. Prediction of turbulent rough-wall skin friction using a discrete element approach. Journal of Fluids Engineering. 1985 June;107:251-257.

[31] Coleman H, Hodge B, Taylor R. A re-evaluation of Schlichting's surface roughness experiment. Journal of Fluids Engineering. 1984 March;106:60-65.

[32] Taylor R, Scaggs W, Coleman H. Measurement and prediction of the effects of nonuniform surface roughness on turbulent flow friction coefficients. Journal of Fluids Engineering. 1988 December; $110: 380-384$.

[33] Bons J, McClain S. The effect of real turbine roughness with pressure gradient and heat transfer. Journal of Turbomachinery. 2004 July;126:385-394.

[34] McClain S, Hodge B, Bons J. Predicting skin friction and heat transfer for turbulent flow over real gas turbine surface roughness using the discrete element method. Journal of Turbomachinery. 2004 April;126:259-267.

[35] McClain S, Collins S, Hodge B, et al. The importance of the mean elevation in predicting skin friction for flow over closely packed surface roughness. Journal of Fluids Engineering. 2006 May;128:579-586.

[36] Aupoix B. Revisiting the discrete element method for predictions of flows over rough surfaces. Journal of Fluids Engineering. 2016 March;:31205. 
[37] Whitaker S. Flows in porous media I: A theoretical derivation of Darcy's law. Transport in Porous Media. 1986;1:3-25.

[38] Whitaker S. The method of volume averaging. Theory and applications of transport in porous media. Vol. 13. Kluwer Academic; 1999.

[39] Hanson DR, Kinzel MP, McClain ST. Validation of the discrete element roughness method for predicting heat transfer on rough surfaces. International Journal of Heat and Mass Transfer. 2019 jun;136:1217-1232. Available from: https://doi.org/10.1016/j.ijheatmasstransfer.2019.03.062.

[40] Kuwata Y, Suga K, Kawaguchi Y. An extension of the second moment closure model for turbulent flows over macro rough walls. International Journal of Heat and Fluid Flow. 2019 Jun;77:186-201. Available from: https://doi.org/10.1016/j.ijheatfluidflow.2019.04.003.

[41] Tarada F. Heat transfer to rough turbine blading [dissertation]. University of Sussex; 1987.

[42] Stripf M, Schulz A, Bauer HJ. Modeling of rough-wall boundary layer transition and heat transfer on turbine airfoils. Journal of Turbomachinery. 2008 Feb;130(2). Available from: https://doi.org/10.1115/1.2750675.

[43] Stripf M, Schulz A, Bauer H, et al. Extended models for transitional rough wall boundary layers with heat transfer - Part I: Model formulations. Journal of Turbomachinery. 2009 July;131:031016-1 - 031016-10.

[44] Stripf M, Schulz A, Bauer H, et al. Extended models for transitional rough wall boundary layers with heat transfer - Part II: Model validation and benchmarking. Journal of Turbomachinery. 2009 July;131:031017-1 - 031017-11.

[45] Kuwata Y, Kawaguchi Y. Direct numerical simulation of turbulence over resolved and modeled rough walls with irregularly distributed roughness. International Journal of Heat and Fluid Flow. 2019 Jun;77:1-18. Available from: https://doi.org/10.1016/j.ijheatfluidflow.2019.02.009.

[46] Forooghi P, Stripf M, Frohnapfel B. A systematic study of turbulent heat transfer over rough walls. International Journal of Heat and Mass Transfer. 2018 dec;127:1157-1168.

[47] Whitaker S. The forchheimer equation: A theoretical development. Transport in Porous Media. 1996;25:27-61.

[48] Jelly TO, Busse A. Reynolds number dependence of reynolds and dispersive stresses in turbulent channel flow past irregular near-gaussian roughness. International Journal of Heat and Fluid Flow. 2019 Dec;80:108485. Available from: https://doi.org/10.1016/j.ijheatfluidflow.2019.108485.

[49] Kuwata Y, Kawaguchi Y. Direct numerical simulation of turbulence over systematically varied irregular rough surfaces. Journal of Fluid Mechanics. 2019 Jan;862:781-815. Available from: https://doi.org/10.1017/jfm.2018.953.

[50] Forooghi P, Stroh A, Magagnato F, et al. Toward a universal roughness correlation. Journal of Fluids Engineering. 2017 aug;139(12):121201.

[51] Taylor R, Coleman H, Hodge B. Prediction of heat transfer in turbulent flow over rough surfaces. Journal of Heat Transfer. 1989 May;111:568-572.

[52] Hanson D. Computational investigation of convective heat transfer on ice-roughened aerodynamic surfaces [dissertation]. The Pennsylvania State University: Aerospace Engineering; 2017.

[53] Forooghi P, Weidenlener A, Magagnato F, et al. DNS of momentum and heat transfer over rough surfaces based on realistic combustion chamber deposit geometries. International Journal of Heat and Fluid Flow. 2018 feb;69:83-94.

[54] Chevalier M, Schlatter P, Lundbladh A, et al. Simson-a pseudo-spectral solver for incompressible boundary layer flow. 2007;Tech. Report no. TRITA-MEK 2007:07.

[55] Goldstein D, Handler R, Sirovich L. Modeling a no-slip flow boundary with an external force field. J Comput Phys. 1993;105(2):354-366.

[56] Forooghi P, Frohnapfel B, Magagnato F, et al. A modified parametric forcing approach for modelling of roughness. International Journal of Heat and Fluid Flow. 2018 jun;71:200 
209.

[57] Antonialli LA, Silveira-Neto A. Theoretical study of fully developed turbulent flow in a channel, using prandtl's mixing length model. Journal of Applied Mathematics and Physics. 2018;06(04):677-692. Available from: https://doi.org/10.4236/jamp.2018.64061.

[58] Raupach M, Shaw R. Averaging procedures for flow within vegetation canopies. Boundary Layer Meteorology. 1982;22:79-90.

[59] Squire D, Morrill-Winter C, Hutchins N, et al. Comparison of turbulent boundary layers over smooth and rough surfaces up to high reynolds numbers. Journal of Fluid Mechanics. 2016; $795: 210-240$.

[60] Jackson P. On the displacement height in the logarithmic velocity profile. Journal of Fluid Mechanics. 1981;111:15-25.

[61] Charru F, Andreotti B, Claudin P. Sand ripples and dunes. Annual Review of Fluid Mechanics. 2013 Jan;45(1):469-493. Available from: https://doi.org/10.1146/annurev-fluid011212-140806.

[62] Claudin P, Durán O, Andreotti B. Dissolution instability and roughening transition. Journal of Fluid Mechanics. 2017 Oct;832. Available from: https://doi.org/10.1017/jfm.2017.711.

[63] Schlichting H. Experimental investigation of the problem of surface roughness. Washington: NACA; 1937. Technical Memorandum 823.

[64] Perry A, Schofield W, Joubert P. Rough wall turbulent boundary layers. Journal of Fluid Mechanics. 1969;37:383. 